\title{
L'elaborazione dei dati computerizzati come ausilio alle decisioni medico infermieristiche
}

\author{
D. Castelnuovo, G. Bacchini, G. Pontoriero, V. Pozzoni, M. Todeschini, M. Andreoli \\ Divisione di Nefrologia e Servizio di Dialisi Ospedale di Lecco
}

1 crescente numero della popolazione dialitica ha portato a gestire una grossa mole di dati da archiviare (1985: 170, 1994: 340 pazienti), memorizzare, elaborare costringendo a dedicare buona parte del tempo destinato all'assistenza medico infermieristica a procedure manuali di trascrizione dei dati su supporti tradizionali quali cartelle cliniche, schedari e archivi vari. Sotto l'impulso di esigenze sempre più complesse e varie il nostro centro ha guardato all'informatizzazione come punto di svolta; quindi dal 1990 il centro ha addottato una lenta ma proficua politica di informatizzazione coinvolgendo tutto lo staff sanitario (medici, infermieri, tecnici, amministrativi).

Superata la prima fase di disorientamento in cui il computer veniva visto come un mezzo nuovo e a volte improprio per l'ambito sanitario, si assiste ora ad una velocizzazione di sintesi dei dati stessi, con notevole vantaggio per il tempo reale dedicato all'assistenza e alla cura del paziente. La concentrazione e la riduzio- ne degli spazi ottenuta con l'informatizzazione agevola la velocità all'accesso dei dati clinici e anagrafici a tutte le più svariate informazioni sui nostri pazienti. La nostra cartella clinica computerizzata è composta da 20 fogli elettronici (Fig. 1) strutturati per avere al suo interno una parte dedicata alle notizie anagrafiche, una parte per i dati clinici e una parte a tutti quei dati che servono per la compilazione del registro regionale. Il software da noi utilizzato è il "Clinix" che altro non è che un Databa-

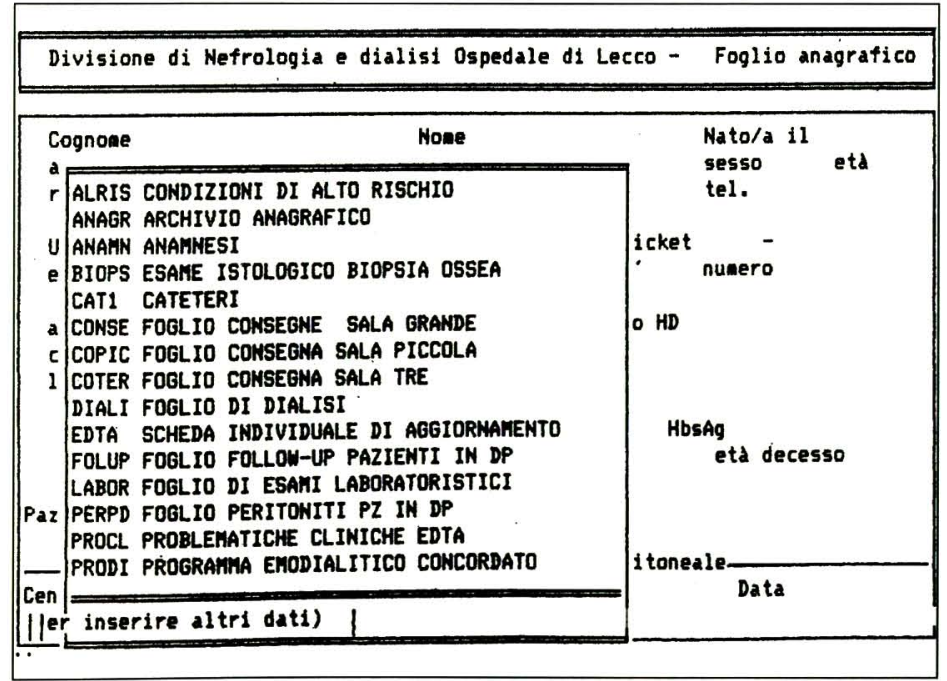

Fig. 1 - Rene destro con i caratteri ecotomografici del rene atrofico.

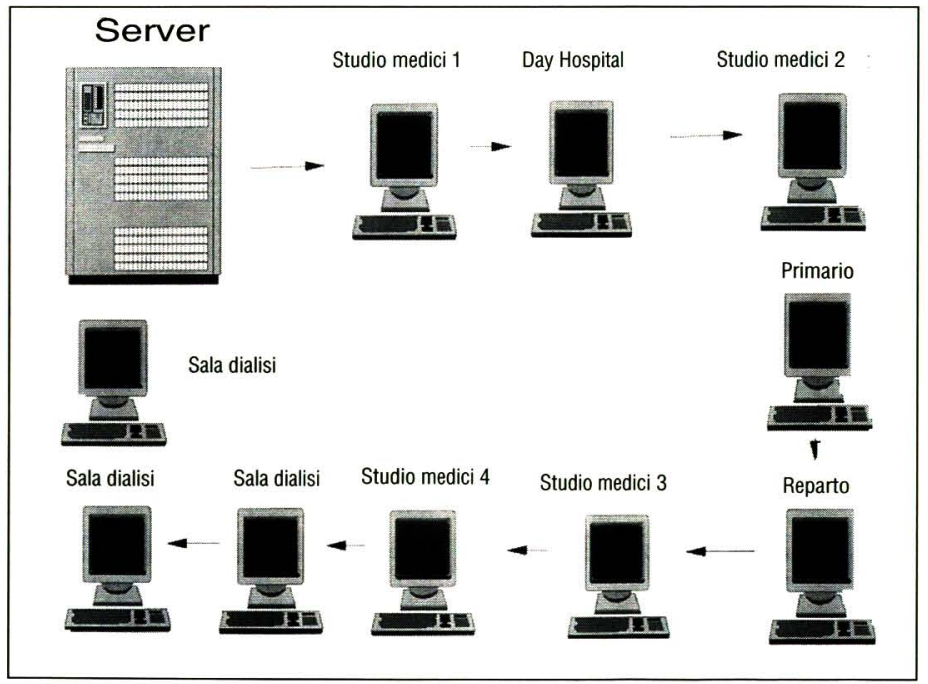

Fig. 2 
TAB. I - CARATTERISTICHE TECNICHE HARDWARE

\begin{tabular}{lcccc}
\hline & Processore & Clock & Ram & S. operativo \\
Server & $486 \mathrm{DX} 2$ & 66 & $12 \mathrm{Mb}$ & Novell 3.12 \\
Stazioni & $486 \mathrm{SX}$ & 33 & $8 \mathrm{Mb}$ & MSdos 6.22 \\
\hline
\end{tabular}

se relazione multimediale. La scelta per questo programma è stata dettata per le sue caratteristiche peculiari quali: versatilità, duttilità e soprattutto semplicità d'utilizzo.
Il nostro sistema informatico è costituito da 12 stazioni (Fig. 2) tutte comunicanti fra loro attraverso una rete locale (Novell 3.12) (Tab. I).

Il nostro centro può altresì comunicare

Fig. 3

\section{Divisione di Nefrologia e Servizio di dialisi \\ Ospedale di Lecco \\ Primario Prof. F. Locatelli}

Si dichiara che il Sig. Rossi Paolo nato a Lecco il 1/05/58, in trattamento emodialitico cronico per IRC nel mese di Aprile 1995 ha effettuato in regime ambulatoriale presso il servizio di dialisi dell'Ospedale di Lecco le seguenti sedute di dialisi extracorporea:

\section{$02 / 04 / 95$}

$05 / 04 / 95$

$07 / 04 / 95$

$09 / 04 / 95$

$12 / 04 / 95$

$14 / 04 / 95$

$16 / 04 / 65$

$19 / 04 / 95$

$21 / 04 / 95$

23/04/95

$26 / 04 / 95$

Si rilascia il presente certificato ai fini del rimborso delle spese di trasporto per il trattamento emodialitico (Legge regionale 5 Novembre 1993, n. 36 , art. 4) via modem con i computer dislocati anche nei centri ad assistenza limitata (Bellano, Oggiono, Merate) avendo così a disposizione in tempo reale anche $\mathrm{i}$ dati di quest'ultimi.

L'utilizzo dei computer ci ha permesso di produrre testi standard (Fig. 3) che possono essere stampati un'infinità di volte senza perdere l'unicità e la possibilità di personalizzazione. Possiamo elaborare noi stessi delle interrogazioni (queries) le quali possono essere memorizzate e richiamate velocemente con un tempo variabile da alcuni secondi ad un massimo di 5 minuti circa. Grazie a queste interrogazioni, molte procedure routinarie del lavoro in sala dialisi, come ad esempio la preparazione del turno dialisi, la pianificazione e organizzazione della sala, vengono effettuate tramite computer a video.

Abbiamo altresì realizzato, essendo la nostra cartella informatica un chiaro esempio di cartella unica medico infermieristica, la standardizzazione e la prescrizione della terapia postdialitica a video, evitando quindi gli inevitabili errori che le ripetute e numerose trascrizioni generano.

E stato possibile anche raccogliere tutti i dati anamnestici, laboratoristici e clinici dei pazienti in trattamento dialitico, richiesti per la compilazione delle schede regionali, stampate quindi in seguito con report ad hoc, dal nostro sistema informativo.

In fase di realizzazione è ora l'implementazione della nostra rete locale con le esistenti reti compartimentali della struttura ospedaliera a completamento del progetto di informatizzazione generale. 